\title{
Environmental surface contamination with SARS- CoV-2 - a short review
}

\begin{abstract}
A highly contagious virus causing an illness called COVID-19 has rapidly emerged in the last four months. The growing demand for intensive healthcare, a rising death toll and disruption to supply chains and trade has led to mass implementation of testing, quarantine and lockdown in an effort to limit virus transmission. There are two ways to contract the virus: person-to-person (direct contact) or indirect (non-contact) transmission. This paper examines what is known to date about non-contact transmission in the built environment in the form of a mini review. The main takeaway message is that high-touch surfaces, toilets and often over-looked objects and surfaces are virus reservoirs and that transmission almost always occurs indoors. The use of reverse transcriptase polymerase chain reaction surveillance (RT-PCR, qPCR) in combination with careful or improved hand hygiene practice and regular surface disinfection cleaning can reduce the environmental viral burden and should not be overlooked or given preference over social distancing interventions.
\end{abstract}

Volume 8 Issue I - 2020

\author{
Cameron L Jones ${ }^{1,2}$ \\ 'Biological Health Services, Australia \\ ${ }^{2}$ National Institute of Integrative Medicine, Australia
}

\begin{abstract}
Correspondence: Dr. Cameron L Jones, Biological Health Services, Level I, 459 Toorak Rd, Toorak, Victoria, 3|42, Australia, Tel+61414998900, Email info@biologicalhealthservices.com.au
\end{abstract}

Received: April II, 2020 | Published: April 27, 2020

Keywords: SARS-CoV-2, COVID-19, fomites, environmental cleaning, surface contamination, non-contact transmission, environmental screening, surveillance, disinfection, RT-PCR, qPCR

\section{Introduction}

Severe acute respiratory syndrome coronavirus (SARS-CoV-2) is the causative agent of the emerging pandemic of 2019 novel coronavirus disease (COVID-19). Tracking the dynamics of this newly emerging outbreak is an important public health challenge. In turn, the surveillance effort has looked at factors from initial exposure, to symptom onset, to medical intervention and hospitalization, to recovery or death. A better understanding of different aspects at each of these stages has significant impacts for intervention strategies. To date, non-pharmaceutical interventions have included contact tracing, quarantine and social distancing. A heightened awareness of general infection control principles has also emerged.

Exposure to SARS-CoV-2 occurs through direct and indirect contact with symptomatic and asymptomatic carriers who shed the virus. Person-to-person or contact transmission is easy to understand with well-developed public health messaging surrounding travel, coughing, sneezing and hand washing. However, contact with objects and surfaces likely to carry infection (fomites) within the environment is less easy to visualize as part of messaging, except as a generalized problem, and mainly as this relates to hand washing. In the literature this is often called non-contact transmission. ${ }^{1}$

The purpose of this paper is to provide a summary of the chronology of publications between February and mid-April 2020 that have examined viral surface contamination within the built environment, including where positive surfaces were detected as well as any survival data. This information can then be used to provide better guidance for surveillance and contact surface testing inside buildings. In turn, this approach could enhance the effectiveness of environmental cleaning and reduce risk from inadvertent exposure with contaminated surfaces.

\section{Methods}

A search was made for the terms "COVID-19" OR "SARS-CoV-2" AND "surfaces" OR "fomites" OR "non-contact" on PubMed, the Cochrane library and the medRxiv preprint server. This yielded 23 articles on PubMed, 305 on medRixv and none on the Cochrane library. Subsequently the abstracts were reviewed and overlap, duplicate or repeating documents identified and removed to create a benchmark dataset. This was used to determine if they were related to surface or fomite contamination as opposed to person-to-person contact spread. Ultimately, we ended up with 16 articles that provided information about either search term and environmental contamination with a specific focus on surfaces. It was noted that at the time, literature on the topic is growing rapidly, and the search results were dominated by publications on the medRixv preprint server and as such it should be acknowledged that many of these are awaiting peer review.

\section{Results}

We included 16 published literature references related to surface contamination and non-contact transmission or testing of SARS$\mathrm{CoV}-2$ in the environment.

1. The first paper discussing SARS-CoV-2 reviewed 22 studies from the literature about the persistence of human and animal coronaviruses as well as inactivation using chemical disinfectants. ${ }^{2}$. They found that SARS and Middle East Respiratory Syndrome (MERS), coronavirus or endemic human coronaviruses can persist on surfaces like metal, glass or plastic for up to 9 days. Virus inactivation was achieved within 1 minute using $62-71 \%$ ethanol, $0.5 \%$ hydrogen peroxide or $0.1 \%$ sodium hypochlorite; while $0.05-0.2 \%$ benzalkonium chloride or $0.02 \%$ chlorhexidine digluconate were less effective. The 
authors concluded that without specific therapies for COVID-19, early containment and prevention of spread is crucial to control infection.

2. In early March, hospital environmental hygiene was assessed ${ }^{3}$ in Changchun, China which is over $1800 \mathrm{~km}$ to the North of Wuhan, considered the origin of the virus. Surface sampling specific for SARS-CoV-2 in the morning before disinfection returned positive results at the nurses' station keyboard (16.67\%) from the isolation ward, but across 129 other surface samples, only 1 tested positive $(0.77 \%)$. Surface monitoring was negative for door handles, toilet handles and personal protective clothing (PPE) worn by medical staff. The overall level of surface contamination was $0.77 \%$ and this was in all cases linked to computer keyboards that were considered to be easily overlooked items during routine cleaning. Reasons for low surface levels may be linked to the use at this hospital of $24 \mathrm{hr}$ ultraviolet germicidal air filtering and use of chlorine-based disinfectant for both air and surface disinfection.

3. A short research letter by Ong et al. ${ }^{4}$ evaluated the extent of environmental contamination at a dedicated SARS-CoV-2 outbreak centre in Singapore. Rooms used by three patients at different stages of illness were evaluated before and after routine cleaning. This was performed twice daily using 5000ppm sodium dichlorocyanurate for surfaces or $1000 \mathrm{ppm}$ for floors. Samples taken before cleaning from one of the patients tested different room surfaces. Of these, 13 of 15 surfaces $(87 \%)$ including air outlet fans were positive. Other surfaces from the patient's room, the toilet area, the anteroom and the corridor outside the patient's room were tested. These included the cardiac table, entire length of bed rail, locker with hand slot, chair, light switch behind bed, stethoscope, sink, external rim, sink, internal bowl, floor, glass window in room, glass door interior surface, PPE storage area over sink, door handle, toilet bowl surface, toilet sink, internal bowl, sink internal/external bowl, floor, glass door on room side and corridor side. Combining these areas showed an overall level of SARS-CoV-2 surface contamination of $61 \%$. It was concluded that respiratory droplet spread, and faecal shedding was responsible for environmental contamination.

4. Obvious questions have emerged regarding what PPE should be used for cleaning and disinfection of rooms or areas that have been used by persons determined or suspected of being infected with SARS-CoV-2? In Korea, minimum respiratory protection equivalent to N95 or equal level, disposable gloves, disposable arm-length gown, whole body protective clothing including slippers and eyeglasses, or a face shield are recommended to prevent nosocomial infections. ${ }^{5}$

5. In an effort to better protect healthcare workers, a key question for a hospital in Wuhan was to determine the environmental contamination levels. ${ }^{6}$ Within the hospital, 626 surfaces from 13 zones were tested. The most contaminated objects were selfservice printers $(20 \%)$, hand sanitizer $(20.3 \%)$, gloves $(15.4 \%)$ and doorknobs (16\%). The most contaminated zones: intensive care unit used for COVID-19 pneumonia patients (31.9\%), obstetric isolation ward for pregnant women $(28.1 \%)$ and the isolation ward for SARS-CoV-2 pneumonia (19.6\%).

6. Influenza A and B virus survival was examined in kindergartens and primary schools in Hong Kong during the 2017/18 influenza season. ${ }^{7}$ Although this paper is not on SARS-CoV-2, the focus on common (high-touch) surfaces like doorknobs, desks and chairs, bookshelves, shared toys and walls, staircase handrails, bathroom cubicle locks and faucets builds on other related papers reviewed here and reveals potential hotspots for virus transmission in schools that may extrapolate to SARS-CoV-2. Samples were taken at the beginning of the day prior to cleaning and disinfection of classrooms and included both high-touch and low-touch surfaces like spare desks and chairs and walls at low height. Notably, less than $1 \%$ of classroom samples (1352) tested positive for viral RNA. High touch surfaces showed $0.7 \%$ and low-touch surfaces showed $2.3 \%$. All of the positive results were from plastic, metal or wood surfaces or those with lacquer finishing. Influenza RNA was more frequently detected from bookshelves and doorknobs or doors inside classrooms. The conclusion was that the potential for indirect contact transmission was confirmed, although with a preference for communal items and low-touch surfaces. Interventions following this data include the use of alcohol sanitisers inside classrooms for hand hygiene and improved surface disinfection as part of cleaning.

7. A University medical centre cared for 13 individuals with confirmed SARS-CoV-2 infection. ${ }^{8}$ The purpose of their research was to determine the impact of viral shedding into the local environment (air and surfaces) during isolation. From quarantine rooms: $76.5 \%$ of all personal items tested positive. Personal items included: cell phones, iPads, reading glasses, personal computers, television remote controls, exercise equipment, pots used to heat water, a nasal canula and spirometer, and the rim and seat of the toilet. Of these samples, between $64.7-83.3 \%$ were positive. Toilets were $81 \%$ positive, $75 \%$ of bedside tables and $81.8 \%$ of windowsills. $80 \%$ of the ventilation grates and $100 \%$ of the floors were positive. Typical room surfaces that were positive for SARS-CoV-2 included the windowsill and bedside table, bed rail, under the patient's bed and the air conditioning return grate nearest the door. Disease spread was concluded to occur (person-to-person) via direct droplet transfer as well as through indirect contact with contaminated objects and possibly through airborne transmission.

8. The stability of SARS-CoV-2 and SARS-CoV-1 was evaluated in air and on various surfaces in a controlled experiment. ${ }^{9}$ Viability of SARS-CoV-2 was maintained for at least $3 \mathrm{hrs}$ in the air, which was the duration of the experiment. On surfaces, the predicted decay of SARS-Co-V2 virus titer was more stable on plastic ( $3+$ days) and stainless steel ( 3 days) than on copper ( $<1$ day) or cardboard (1-2 days). Companion testing showed the tissue-culture infectious dose $\left[\mathrm{TCID}_{50}\right]$ per $\mathrm{mL}$, and the stability from highest to lowest above the limit of detection was: Plastic $(72 \mathrm{hrs})>$ Stainless steel $(48 \mathrm{hrs})>$ cardboard $(8 \mathrm{hrs})$ $>$ copper (8hrs). The conclusion was that aerosol and fomite transmission is plausible and that SARS-CoV-2 can survive in the air for hours and for days on surfaces which was similar to the decay curve seen for SARS-CoV-1. For either virus, there was exponential decay over time with survival depending on the amount of inoculum shed.

9. It is estimated that $30 \mathrm{M}$ passengers travel on cruise ships worldwide every year. A report in late March discussed outbreaks of SARS-CoV-2 on several cruise ships including the Diamond Princess and Grand Princess. ${ }^{10}$ This resulted in over 800 
confirmed cases of COVID-19 and more than 10 deaths. Testing for SARS-CoV-2 from a variety of surfaces in cabins taken from both symptomatic and asymptomatic passengers recovered RNA for up to 17 days after the cabins were vacated on the Diamond Princess, but before disinfection. The conclusion was that aggressive efforts are required to contain fomite transmission, although the evidence was inconclusive that transmission to passengers had occurred from contaminated surfaces and that further investigation was warranted.

10. Patient-level risk factors for environmental contamination were evaluated at the National Centre for Infectious Diseases (Singapore), which is a high-level isolation unit." ${ }^{11}$ Sampling occurred at different stages of illness, with 245 surfaces from 30 rooms with COVID-19 patients and air sampling performed in 3 rooms. The results showed that $56.7 \%$ of rooms had at least 1 surface contaminated. The toilet seats and toilet flush button were contaminated in $18.5 \%$ of samples. High touch surface contamination was seen in $66.7 \%$ of patients in the first week of illness, and 20\% after the first week of illness. Environmental cleaning of rooms, and especially high-touch surfaces (bed rail, cardiac table, switches) were cleaned twice daily using 5000ppm sodium dichloroisocyanurate $(\mathrm{NaDCC})$ reconstituted using Biospot ${ }^{\circledR}$ Effervescent Chlorine Tablets. The floor was cleaned daily using $1000 \mathrm{ppm}$ NaDCC. All surface sampling was done in the morning before the first cleaning cycle. Information from the supplementary material showed that the following surfaces were positive for SARS-CoV-2 at some stage of the patients' admission: table, bed rail, locker handle, electric switch, chair, floor, toilet seat and flush handle, air exhaust vent. The conclusion was that concentrations in the air and on high touch surfaces occurred during the first week of illness.

11. There have been many reports of hospitals stretched beyond capacity and of dwindling supplies of PPE. ${ }^{12}$ Their paper summarised the evidence for SARS-CoV-1 survivorship and methods to disinfect PPE equipment and in particular N95 filtering facepiece respirators. This was in an effort to increase the supply of PPE to frontline staff and mitigate the supply chain disruption and extrapolate from what is known about SARS-CoV-1 to SARS-CoV-2. A 2-step disinfection protocol was developed. Initial storage of potentially or known to be contaminated PPE for $\geq 4$ days, followed by exposure to ultraviolet germicidal irradiation (UVC), dry heat or chemical disinfection. This protocol was based on a review of the literature reporting survival of SARS-CoV-1 where variously: Wood board and mosaic (4 days), glass, press paper, plastic, water, soil (5 days), metal cloth, filter paper (5 days), serum, filtered sputum (4 days), sputum, faeces, filtered faeces, urine (5 days), a disposable polypropylene gown (2 days), a cotton gown (1 day), respiratory specimens at room temperature (9 days), a plastic petri plate ( 9 days), hospital wastewater, domestic sewage, dechlorinated tap water (3-14 days), stool (4 days), urine (17 days). It was concluded that until more data becoming available for SARS-CoV-2, it should be assumed that the newer SARS virus may survive for longer than SARS-CoV-1.

12. The issue of adverse air quality and its' impact on COVID-19 infections has recently been reported, ${ }^{13}$ where it was shown that higher mortality was correlated with poor air quality indexed with $\mathrm{PM} 2.5, \mathrm{CO}$ and $\mathrm{NO} 2$. The conclusion was that people exposed to low air quality, and especially particulate matter pollution, were more vulnerable to SARS-CoV-2 infections and that this variable was overlooked with respect to virus transmission, despite the strong correlation with other respiratory diseases.

13. A report from China reviewed what is known about the transmission of SARS-CoV-2 and examined outbreaks from case reports across 320 cities, not including Hubei province over 41 days spanning January-February. ${ }^{14}$ Out of 1245 cases in 120 cities, the outbreak size distribution showed that $53.8 \%$ involved 3 cases, $26.4 \%$ had 4 cases and only $1.6 \%$ had more than 10 cases. Outbreaks at home were the dominant category at $79.9 \%$, followed by transport at $34 \%$ and $4.4 \%$ at a restaurant or food outlet, $2.2 \%$ at an entertainment venue, $2.2 \%$ as a shopping mall or supermarket and $8.18 \%$ at a miscellaneous venue (hospital, hotel room, community or thermal power plant). There was overlap between categories. Large outbreaks were highest for shops and food venues compared with homes. There was only a single outbreak from the outdoor environment.The conclusion was that crowding in shared indoor spaces is the major infection risk and that effort should focus on the provision of improved indoor air quality.

14. Extensive sampling at a dedicated COVID-19 hospital in Nanjing, which is to the North-East of Wuhan looked at the faecal-oral route and the potential for aerosolization into the air and onto surfaces. ${ }^{15}$ The following room types were sampled: isolation rooms, a nursing station, corridor, an air conditioning system over one-floor of this 6-story hospital. Positive surfaces included the ward door handle (ward-side), bathroom door handle, bathroom ceiling exhaust louvre, washbasin faucet lever, toilet seat, and the lower surface of the toilet seat cover. The summary results showed positive samples from $13.5 \%$ of toilet related surfaces and 5.9\% from other surfaces within isolation rooms. It was noted that a limitation of the study was the fact the sampling operative was only permitted to test in rooms with patients having mild symptoms. The conclusion was that toilets are high risk areas in hospitals with COVID-19 patients and that both public and private toilets should be carefully managed with respect to hand and environmental hygiene.

15. The issue of infection control and prevention in non-clinical areas was reported on for a hospital in Hong Kong, ${ }^{16}$ and was considered a neglected area at high risk for transmission. Interventions consisted of alcohol hand sanitizer placed in each office, changing room, department entrance, computer terminals and communal areas. The keyboards and doorway keypads were wrapped in transparent covers and cleaned regularly. All partitions, water fountains, tables and common areas were regularly cleaned with a chlorine-based disinfectant (Actichlor ${ }^{\mathrm{TM}}$ at $1000 \mathrm{ppm}$ ) before and after use.

16. The stability of SARS-CoV-2 has been simulated under various environmental conditions in order to help with optimizing standard disinfection methods. ${ }^{17}$ The virus was highly stable at $4^{\circ} \mathrm{C}$ but was inactivated at $70^{\circ} \mathrm{C}$ after $5 \mathrm{~min}$. If a small $5 \mu \mathrm{L}$ droplet containing virus was inoculated onto various surfaces, then no infectious virus could be recovered on paper or tissue paper after $3 \mathrm{hrs,} \mathrm{wood} \mathrm{and} \mathrm{cloth} \mathrm{after} 2$ days, glass and banknotes after 4 days, stainless steel, plastic and the inner layer of masks after 7 days but the outer layer of masks remained infectious even after 7 days. 


\section{Discussion}

Indirect or non-contact transmission of SARS-CoV-2 is the other infection pathway that doesn't involve direct, person-to-person contact. The potential for widespread environmental contamination and the need for targeted remediation is hence considered an enormous challenge. To date, most approaches aim to reduce contact transmission through active surveillance, contact tracing, quarantine, social distancing and lockdown. ${ }^{18}$ Interestingly, epidemiological modelling of fomite transmission dynamics ${ }^{19}$ showed that to make lockdown effective, there is a need to reduce non-contact transmission. Lowering the non-contact transmission rate was key to bringing down the number of infection cases below the hypothetical healthcare capacity. It was therefore recommended that active measures to reduce air and surface contamination should occur and that any premature withdrawal of lockdown could allow re-activation of either of the two transmission routes. The conclusion was that any exit policy should take into account transmission reduction for both pathways.

Before SARS-CoV-2, modelling efforts aimed at capturing the transmission dynamics of norovirus and other microorganisms have shown that hand hygiene alone is insufficient as a control measure for limiting fomite transmission of pathogens ${ }^{19}$ and that surface cleaning frequency depends on both the survival ability of the pathogen and on the number and frequency of hand-to-surface contacts. Indeed, earlier work with norovirus showed that hand contact could easily transfer to surfaces like taps, door handles, telephone receivers and melamine; and that contaminated hands could sequentially transfer virus to up to seven clean surfaces. ${ }^{20}$ Other models have focused on occupational health in communal offices by looking at (single and combined) interventions for personal hand hygiene and surface disinfection. The results showedthe combined approach led to an $87.8 \%$ reduced infection risk for common viruses like rotavirus, rhinovirus and influenza. ${ }^{21}$ In practice, viral transmission in the workplace can be visualized using a safe, ethical MS2 phage tracer that does not infect humans but can be applied to high-touch building elements like door handles. This allows fomite transmission to be tracked and not just modelled. In this study, surfaces found to be most contaminated were the refrigerator, drawer handles, sink faucets in the break room, the pushbar on the main exit to the building, communal computer station, desk chair arms and soap dispensers in the women's restrooms. ${ }^{22}$

Screening for SARS-CoV-2 involves swabbing suspect items or surfaces and is based on the polymerase chain reaction (PCR) used to test clinical specimens like those from the back of the nose to diagnose people for COVID-19. The method is based on increasing the number of copies of a target gene in order to help determine if a particular DNA fragment is present. A positive result is seen when viral genetic material is amplified and is detected as a fluorescent signal. When no viral genetic material is present in a sample, then amplification doesn't occur, and no fluorescence is detected. ${ }^{23}$ The molecular principles of PCR have been developed for SARS-associated coronavirus detection using the real-time reverse transcription polymerase chain reaction (RT-PCR $)^{24}$ which uses RNA as the template molecule. For example, detecting viruses in the urban environment like the New York subway on doors or inside trains is called environmental sequencing and exploits this PCR reaction ${ }^{25}$ or variants like quantitative PCR (qPCR), and can obviously be extended to any other region or locale of interest.

Once surfaces have been confirmed to be contaminated or the probability of contamination with SARS-CoV-2 is high, then environmental cleaning and disinfection becomes mandatory.
Guidance on these steps have been provided by the US EPA in the List $\mathrm{N}$ for chemical disinfectants ${ }^{26}$ and by the $\mathrm{CDC}^{27}$ and the Australian Department of Health. ${ }^{28}$

\section{Conclusion}

SARS-CoV-2 is an emergent and highly contagious virus which is disrupting lives and the economy. ${ }^{29}$ Urgent measures are therefore needed to contain and manage the pandemic to save lives and help move towards economic stability. Environmental infection control of the air and especially for surfaces is considered a mandatory step in addition to limiting person-to-person contact. To this end, the role of environmental surveillance, site inspections and audits and cleaning is more important than ever.

\section{Funding}

No funding.

\section{Acknowledgments}

None.

\section{Conflicts of interest}

The author declares no conflictof interest.

\section{References}

1. Roy S. COVID-19 pandemic: Impact of lockdown, contact and noncontact transmissions on infection dynamics. medRxiv preprint. 2020.

2. Kampf G, Todt D, Pfaender S, et al. Persistence of coronaviruses on inanimate surfaces and their inactivation with biocidal agents. $J$ of Hosp Infect. 2020;104(3):246-251.

3. Jiang Y, Wang H, Chen Y, et al. Clinical Data on Hospital Environmental Hygiene Monitoring and Medical Staff Protection during the Coronavirus Disease 2019 Outbreak. medRxiv preprint. 2020.

4. Ong S, Tan Y, Chia P, et al. Air, Surface Environmental, and Personal Protective Equipment Contamination by Severe Acute Respiratory Syndrome Coronavirus 2 (SARS-CoV-2) From a Symptomatic Patient. JAMA. 2020.

5. Huh S. How to train health personnel to protect themselves from SARS-CoV-2 (novel coronavirus) infection when caring for a patient or suspected case. J Educ Eval Health Prof. 2020;17:10.

6. Ye G, Lin H, Chen L, et al. Environmental contamination of the SARSCoV-2 in healthcare premises: An urgent call for protection for healthcare workers. medRxiv preprint. 2020.

7. Fong MW, Leung NHL, Xiao J, et al. Presence of influenza virus on touch-surfaces in kindergartens and primary schools [published online ahead of print, 2020 Mar 16]. J Infect Dis. 2020;jiaa114.

8. Santarpia J, Rivera D, Herrera V, et al. Transmission Potential of SARSCoV-2 in Viral Shedding Observed at the University of Nebraska Medical Center. medRxiv preprint. 2020.

9. van Doremalen N, Bushmaker T, Morris D, et al. Aerosol and surface stability of HCoV-19 (SARS-CoV-2) compared to SARS-CoV-1. medRxiv preprint. 2020.

10. Moriarty LF, Plucinski MM, Marston BJ, et al. Public Health Responses to COVID-19 Outbreaks on Cruise Ships - Worldwide, February-March 2020. MMWR Morb Mortal Wkly Rep. 2020;69:347-352.

11. Chia P, Coleman K, Tan Y, et al. Detection of Air and Surface Contamination by Severe Acute Respiratory Syndrome Coronavirus 2 (SARS-CoV-2) in Hospital Rooms of Infected Patients. medRxiv preprint. 2020 
12. Derraik J, Anderson W, Connelly E, et al. Rapid evidence summary on SARS-CoV-2 survivorship and disinfection, and a reusable PPE protocol using a double-hit process. medRxiv preprint. 2020.

13. Pansini R, Fornacca D. Initial evidence of higher morbidity and mortality due to SARS-CoV-2 in regions with lower air quality. medRxiv preprint. 2020.

14. Qian H, Miao T, LIU L, et al. Indoor transmission of SARS-CoV-2. medRxiv preprint. 2020

15. Ding Z, Qian H, Xu B, et al. Toilets dominate environmental detection of SARS-CoV-2 virus in a hospital. medRxiv preprint. 2020.

16. Ling L, Wong W, Wan W, et al. Infection control in non-clinical areas during COVID-19 pandemic. Anaesthesia. 2020.

17. Chin A W H, Chu J T S, Perera M R A, et al. Stability of SARS-CoV-2 in different environmental conditions. Lancet Microbe. 2020.

18. Sanche S, Lin Y, Xu C, et al. High Contagiousness and Rapid Spread of Severe Acute Respiratory Syndrome Coronavirus 2. Emerging Infect Dis. 2020;26(7).

19. Lei H, Xiao S, Cowling BJ, et al. Hand hygiene and surface cleaning should be paired for prevention of fomite transmission. Indoor Air. 2019;00:1-11.

20. Barker, J, Vipond, IB, Bloomfield SF. Effects of cleaning and disinfection in reducing the spread of Norovirus contamination via environmental surfaces. J Hosp Infect. 2004;58(1):42-49.
21. Contreras RD, Wilson AM, Garavito F, et al. Assessing virus infection probability in an office setting using stochastic simulation. J Occup Environ Hyg. 2020;17(1):30-37.

22. Kurgat EK, Sexton JD, Garavito F, et al. Impact of a hygiene intervention on virus spread in an office building. International Journal of Hygiene and Environmental Health. 2019.

23. Hadaya J, Schumm M, Livingston EH. Testing Individuals for Coronavirus Disease 2019 (COVID-19). JAMA. 2020.

24. Emery SL, Erdman DD, Bowen MD, et al. Real-time reverse transcriptionpolymerase chain reaction assay for SARS-associated coronavirus. Emerg Infect Dis. 2004;10(2):311-316.

25. Cumbers J. Is Coronavirus In The NYC Subway? How Swabbing Doors And Trains Is Shaping The Fight Against COVID-19.

26. List N: Disinfectants for Use Against SARS-CoV-2 | US EPA. US EPA

27. Coronavirus Disease 2019 (COVID-19) Environmental Cleaning and Disinfection Recommendations. Centers for Disease Control and Prevention 2020.

28. Environmental cleaning and disinfection principles for health and residential care facilities. Health.gov.au. 2020.

29. Monthly Briefing: COVID-19 disrupting lives, economies and societies. Economic Analysis \& Policy Division | Dept of Economic \& Social Affairs | United Nations. 2020. 